\title{
Dynamic Optimization Technique for Distribution of Goods with Stochastic Shortages
}

\author{
Charles I. Nkeki \\ Department of Mathematics, Faculty of Physical Sciences, University of Benin, P.M.B. 1154, Benin City, Edo State, Nigeria \\ Correspondence should be addressed to Charles I. Nkeki; nkekicharles2003@yahoo.com
}

Received 2 April 2013; Accepted 24 September 2013

Academic Editor: Eric S. Fraga

Copyright (C) 2013 Charles I. Nkeki. This is an open access article distributed under the Creative Commons Attribution License, which permits unrestricted use, distribution, and reproduction in any medium, provided the original work is properly cited.

\begin{abstract}
This work considers the distribution of goods with stochastic shortages from factories to stores. It is assumed that in the process of shipping the goods to various stores, some proportion of the goods will be damaged (which will lead to shortage of goods in transit). The cost of the damaged goods is added to the cost of the shipment. A proportion of the total expected cost of the shortage goods is assumed to be recovered and should be deducted from the total cost of the shipment. In order to determine the minimum transportation costs for the operation, we adopt dynamic optimization principles. The optimal transportation cost and optimal control policies of shipping the goods from factories to stores were obtained. We find that the optimal costs of the goods recovered could be determined. It was further found that the optimum costs of distributing the goods with minimum and maximum error bounds coincide only at infinity.
\end{abstract}

\section{Introduction}

A distribution company plans to minimize the cost of distributing $q$ kinds of products from $m$ number of factories to $n$ number of stores. For the case of a company distributing a particular product from one factory to all the stores (a single product), that is, at factory $S_{1}$, product $A$ is distributed to all the $n$ stores and factory $S_{2}$ produce $B$ products and are distributed to all the $n$ stores, and so on; see Nwozo and Nkeki [1]. It is also expected that the goods that leave the factories will not come back to the factories (in the case of defective, damaged, etc., items). The company considered $n$ number of control policies $\mu=\left\{\pi^{1}, \pi^{2}, \ldots, \pi^{n}\right\}$ to determine which of them will yield the optimum control policy. They also estimated that certain percentage of the products are to reach their final destination successfully at minimum costs. It is assumed that some proportion of the shortage goods should be recovered. The recovered costs of the shortage goods should be deducted from the total costs continuously over time horizon.

In the related literature, Powell [2] considered the problem of a stochastic fleet assets management problem and used the postdecision state variable implicitly to determine this problem. Closely related to this on the extensive work on stochastic fleet assets management problem is the work by Cheung and Powell [3]. Godfrey and Powell [4] further used postdecision state variable explicitly to determine similar problem on a stochastic fleet management problem. Van Roy et al. [5] proposed the idea of using a parsimonious sufficient statistics in an application of approximate dynamic programming to inventory management. Mulvey and Vladimirou [6] used the stochastic programming technique in financial asset allocation problems for designing low-risk portfolios, and Mulvey and Ruszczynski [7] designed variety of specialized algorithms that help to solve the problems which involved management of homogeneous resources. They further emphasized that for more realistic problems, resources are heterogeneous and that the resulting optimization problems do not generally exhibit pure network structure. Powell et al. [8] described an algorithm for computing parameter values to fit linear and separable concave approximation to the value function to tackle large-scale problems in transportation and logistics. Furthermore, Powell and Topaloglu [9] described a more complicated variation of the algorithm that improved on execution time and memory requirements and such improvement is often critical for practical application to 
realistic large-scale problems. They further emphasized that the movement of freight over long distances is subject to random delays. Powell and Van Roy [10] studied and applied approximate dynamic programming to high-dimensional resource allocation problems in area of managing a fleet of trucks. Powell [22] worked extensively on an approximate dynamic programming for large-scale asset management problems for both single and multiple assets. Guestrin et al. [11] used an approximate dynamic programming approach to deal with high-dimensional decision spaces. For now, no other applications of approximate dynamic programming have dealt with such large numbers of decision variables (see, Powell and Van Roy [10]). Spivey and Powell [12] studied the dynamic assignment problem, where a resource can serve only one task at a time. They further proposed a general class of dynamic assignment models that involved adaptive and nonmyopic algorithm. Cogill et al. [13] considered the problem of computing decentralized policies for stochastic systems with finite state and action spaces. They presented an algorithm based on linear programming and used this algorithm to obtain a decentralized policy from a function with special structure that approximates the optimal centralized Q-function. Topaloglu and Kunnumkal [14] proposed two approximate dynamic programming methods to optimize the distribution operations of a company manufacturing a certain product at multiple production plants and ship to different customer location for sale. Hauskrecht and Singliar [15] considered real-world distributions and networks which are unreliable and subject to random failures of its components. They investigated MonteCarlo solutions in stochastic networks in which the expected value of resources allocated before and after the occurrence of stochastic failures needs to be optimized. Ozbay et al. [16] proposed mathematical programming models with probabilistic constraints in order to address incident response and resource allocation problems for traffic incident management. They considered the resource allocation problem by assuming that the stochastic distribution of incidents over a network is given and introduced a mathematical model to determine the number of service vehicles allocated to each depot to meet the requirements of the potential incidents by taking into account the stochastic nature of the resource requirement and incident occurrence probabilities. Jacko [17] considered resource allocation problem in telecommunication system. They optimized packet losses by choosing preferred paths in the routing system. They assumed that there are a number of independent competitors with the aim of maximizing the expected return. Elmaghraby and Ramachandra [18] considered the problem of optimally allocating a single resource under uncertainty to the various activities of a project to optimize a certain economic objective composed of resource utilization cost and tardiness cost. Nwozo and Nkeki [19], used DPP to consider the allocation of buses from single station to different routes in Nigeria for profit maximization. Nkeki [20] considered the allocation of vehicles from different (multiple) stations to different routes putting into consideration the rate of breakdown of the vehicles during the allocation but failed to put into consideration the depreciation of the vehicles and effect tax on the returns of the investment. Nwozo and
Nkeki [21] studied the allocation of resource to different tasks in a production company so as to maximize returns. They assumed that the company produces the same kinds of products and allocate $m$ number of tasks to $n$ number of machines. They also assumed that the machines are subject to random breakdown.

This paper discussed the distribution of goods from different factories to stores with stochastic shortage over time horizon.

The highlights of this paper are

(a) modeling the distribution of goods from factories to stores in the presence of

(i) stochastic shortages of the goods in transit and

(ii) deterministic case (i.e., when there are no shortages) was also considered, using a dynamic optimization technique,

(b) numerical determination of optimal cost and optimal control policy for the operations was obtained.

The remaining parts of the paper are structured as follows: Section 2 presents the problem formulation and multiperiod expected cost function. In Section 3, we present the dynamic programming formulation of the problem. The optimization of the cost function is determined in this section. Section 4 presents the dynamic programming principles with error bounds. In Section 5, numerical simulations of the problem are discussed. Section 6 concludes the paper.

\section{Problem Formulation}

The paper studies a problem where there are $n$ states and $n$ control policies. Let $S^{\text {factory }}=\left\{S^{f_{1}}, S^{f_{2}}, \ldots, S^{f_{m}}\right\}$ be the set of goods produced in the factories and let $S^{\text {store }}=$ $\left\{S^{s_{1}}, S^{s_{2}}, \ldots, S^{s_{n}}\right\}$ be the set of goods that are to be distributed to the stores, where $S^{\text {factory }}, S^{\text {store }} \in S$ and $S$ is the state space of the system. The transition probability matrix corresponding to the control policies $\pi^{1}, \pi^{2}, \ldots, \pi^{n}$ is given as

$$
P\left(\pi^{j}\right)=P_{i, j}\left(\pi^{j}\right), \quad i=1,2, \ldots, m ; j=1,2, \ldots, n .
$$

The transition costs are given by $\Phi^{i, j}\left(S_{t}^{f_{i}}, \pi_{t}^{j}\left(S_{t}^{f_{i}}\right)\right), t \in[0, T]$, $S_{t}^{f_{i}} \in S$, where $T$ is the terminal time period in the planning horizon, $S_{t}^{f_{i}}$ represents the state of goods produced in factory $i$ at period $t, s_{t}^{s_{j}}$ represents the state of goods to be distributed to store $j$ at period $t$, and the discount factor $\beta,(0 \leq \beta<1)$. We define the function $Q_{t}^{\pi_{t}^{j}}\left(S_{t}^{j}\right)$ as the cost of the distribution of the goods corresponding to the control policies $\pi_{t}^{j}, j=$ $1,2, \ldots, n$. The function $x_{p, t}^{\pi_{t}^{j}}\left(S^{f_{i}}\right)$ represents the number of $p$ different kinds of goods produced in factory $i$ at period $t$ under policy $\pi_{t}^{j}$ and $x_{p, t}^{\pi_{t}^{j}}\left(S^{s_{j}}\right)$ represents the number of $p$ different kinds of goods distributed to store $j$ at period $t$ under the policy $\pi_{t}^{j}$.

2.1. Multiperiod Expected Cost Function. Suppose that the costs of distributing the goods from factory $i$ to store $j$, are 
$\Phi_{t}^{i, j}\left(x_{p, t}^{\pi_{t}^{j}}\left(S_{t}^{f_{i}}\right)\right)$ at period $t$, where the state of goods in factory $i$ is $S_{t}^{f_{i}} \in S_{t}$ at period $t$ and the state of goods to store $j$ is $S_{t}^{s_{j}} \in S_{t}$ at period $t$. Then, the costs over $T$-horizon are

$$
\sum_{t=0}^{T} \sum_{i=1}^{m} \sum_{p=1}^{q} \Phi_{t}^{i, j}\left(x_{p, t}^{\pi_{t}^{j}}\left(S_{t}^{f_{i}}\right)\right), \quad S_{t}^{f_{i}} \in S_{t}, j=1,2, \ldots, n .
$$

Since $x_{p, t}^{\pi_{t}^{j}}\left(S^{f_{i}}\right)$ is the number of $p$ different kinds of goods produced in factory $i$ at period $t$ under policy $\pi_{t}^{j}$ and $x_{p, t}^{\pi_{t}^{j}}\left(S^{s_{j}}\right)$ and the number of $p$ different kinds of goods distributed successfully to store $j$ at period $t$ under policy $\pi_{t}^{j}$, then

$$
\begin{gathered}
\sum_{i=1}^{m} \sum_{p=1}^{q} x_{p, t}^{\pi_{t}^{j}}\left(S^{f_{i}}\right) \geq \sum_{j=1}^{n} \sum_{p=1}^{q} x_{p, t}^{\pi_{t}^{j}}\left(S^{s_{j}}\right), \\
x_{p, t}^{\pi_{t}^{j}}\left(S^{f_{i}}\right), x_{p, t}^{\pi_{t}^{j}}\left(S^{s_{j}}\right) \in X, t \in[0, T] .
\end{gathered}
$$

The expected minimum discounted cost function obtained under control policy $\pi_{t}^{j}$, at period $t$, is given as follows:

$$
\begin{aligned}
& Q_{t}^{\pi_{t}^{j}}\left(S_{t}^{j}\right) \\
& =E_{t}\left(\min _{\substack{\pi_{t}^{j} \\
\pi_{p, t}^{j}\left(S_{i}^{f_{i}}\right), x_{p, t}\left(S_{j}\right) \in X}} \sum_{t=0}^{T} \sum_{i=1}^{m} \sum_{p=1}^{q} \beta^{t} \Phi_{t}^{i, j}\left(x_{p, t}^{\pi_{t}^{j}}\left(S_{t}^{f_{i}}\right)\right)\right), \\
& j=1,2, \ldots, n,
\end{aligned}
$$

subject to

$$
\begin{array}{r}
\sum_{i=1}^{m} \sum_{p=1}^{q} x_{p, t}^{\pi_{t}^{j}}\left(S^{f_{i}}\right) \geq \sum_{j=1}^{n} \sum_{p=1}^{q} x_{p, t}^{\pi_{t}^{j}}\left(S^{s_{j}}\right), \\
x_{p, t}^{\pi_{t}^{j}}\left(S^{f_{i}}\right), x_{p, t}^{\pi_{t}^{j}}\left(S^{s_{j}}\right) \in X, t \in[0, T], \\
\sum_{p=1}^{q} x_{p, t}^{\pi_{t}^{j}}\left(S^{f_{i}}\right) \geq \sum_{p=1}^{q} x_{p, t}^{\pi_{t}^{j}}\left(S^{s_{j}}\right), \\
x_{p, t}^{\pi_{t}^{j}}\left(S^{f_{i}}\right), x_{p, t}^{\pi_{t}^{j}}\left(S^{s_{j}}\right) \in X, t \in[0, T],
\end{array}
$$

where $x_{p, t}^{\pi_{t}^{j}}\left(S^{f_{i}}\right), x_{p, t}^{\pi_{t}^{j}}\left(S^{s_{j}}\right) \in X$ is the set of feasible solutions of problem (4). We can express (4) as the expected discounted minimal cost from period $t$ onward as an optimization over $\left\{x_{p, 1}^{\pi_{1}^{j}}, x_{p, 2}^{\pi_{2}^{j}}, \ldots, x_{p, T}^{\pi_{T}^{j}}\right\}$ condition on $S_{t}^{j}=s_{t}$ as follows:

$$
\begin{aligned}
& Q_{t}^{\pi_{t}^{j}}\left(S_{t}^{j}\right) \\
&=E\left(\operatorname { m i n } _ { \substack { \pi _ { p , 1 } ^ { j } \\
\pi _ { p , 1 } ^ { j } , x _ { p , 2 } ^ { j } , \ldots , x _ { p , T - 1 } ^ { j } } } \left\{\sum_{t^{\prime}=t}^{T} \sum_{i=1}^{m} \sum_{p=1}^{q} \beta^{t^{\prime}} \Phi_{t^{\prime}}^{i, j}\right.\right. \\
&\left.\left.\times\left(x_{p, t^{\prime}}^{\pi_{t}^{j}}\left(S_{t^{\prime}-1}^{j}\right)\right) \mid S_{t}^{j}=s_{t}\right\}\right), \\
& S_{t}^{j} \in S, x_{t} \in X, j=1,2, \ldots, n,
\end{aligned}
$$

subject to the constraints (5).

For a function $\Phi^{i, j} \in \mathbb{R}^{n \times m}, i=1,2, \ldots, m ; j=1,2$, $\ldots, n$, if we accumulate the cost of the first $T$-stage and add to it the terminal cost $\Phi_{T}\left(S_{T}\right)=\sum_{i=1}^{m} \sum_{p=1}^{q} \Phi_{T}^{i, j}\left(x_{p, T}^{\pi_{T}^{j}}\left(S_{T}^{j}\right)\right), j=$ $1,2, \ldots n$, then (6) becomes

$$
\begin{array}{r}
Q_{t}^{\pi_{t}^{j}}\left(S_{t}^{j}\right) \\
=E\left(\operatorname { m i n } _ { x _ { t } } \left\{\sum_{t^{\prime}=t}^{T-1} \sum_{i=1}^{m} \sum_{p=1}^{q} \beta^{t^{\prime}} \Phi_{t^{\prime}}^{i, j}\left(x_{p, t^{\prime}}^{\pi_{t}^{j}}\left(S_{t^{\prime}-1}^{j}\right)\right)\right.\right. \\
\left.\left.+\beta^{T} \Phi_{T}\left(S_{T}^{j}\right) \mid S_{t}^{j}=s_{t}\right\}\right), \\
S_{t}^{j} \in S, x_{p, t}^{\pi_{t}^{j}} \in X, j=1,2, \ldots, n,
\end{array}
$$

subject to the constraints (5).

\section{Dynamic Programming Formulation}

In this section, we formulate the problem using dynamic programming technique. Since $S_{t}$ is the state variable at period $t$ and $S$ the state space, we formulate the problem as a dynamic program. Let the cost of the goods that is to be distributed from factory $i$ to store $j$ at period $t$ under policy $\pi_{t}^{j}$ be given by $P_{i, j} x_{p, t}^{\pi_{t}^{j}}\left(S^{f_{i}}\right)$, where $P_{i, j}$ is the transition probability of the goods from factory $i$ to store $j$. Hence, the total expected cost of the goods distributed from factory $i$ to store $j$ at period $t>0$ is given by

$$
\sum_{i=1}^{m} \sum_{p=1}^{q} P_{i, j} x_{p, t}^{\pi_{t}^{j}}\left(S^{f_{i}}\right), \quad t=1,2, \ldots, T ; j=1,2, \ldots, n .
$$

Let $\gamma$ be the proportion of the total expected cost of goods lost in transit (shortage) from factory $i$ to store $j$. Then, (8) becomes

$$
\gamma \sum_{i=1}^{m} \sum_{p=1}^{q} P_{i, j} x_{p, t}^{\pi_{t}^{j}}\left(S^{f_{i}}\right), \quad t=1,2, \ldots, T ; j=1,2, \ldots, n .
$$


Equation (9) represents the total gross shortage cost incurred from the goods lost in transit.

Let $S_{t-1}^{j}$ be the cost of distributing the goods from the factories to store $j$ in period $t-1$ and $S_{t}^{j}$ the expected cost of distributing the goods from the factories to store $j$ at period $t$ and let $\alpha$ be the estimated proportion of the goods that are to be recovered from the lost ones, which are expected to go to the stores at period $t$, so that the net shortage cost incurred from the goods lost in transit will be

$$
\begin{aligned}
& (1-\alpha) \gamma \sum_{i=1}^{m} \sum_{p=1}^{q} P_{i, j} x_{p, t}^{\pi_{t}^{j}}\left(S^{f_{i}}\right), \\
& t=1,2, \ldots, T ; j=1,2, \ldots, n .
\end{aligned}
$$

It then follows that

$$
\begin{array}{r}
S_{t}^{j}=S_{t-1}^{j}+ \\
\gamma(1-\alpha) \sum_{i=1}^{m} \sum_{p=1}^{q} P_{i, j} x_{p, t}^{\pi_{t}^{j}}\left(S^{f_{i}}\right), \\
j=1,2, \ldots, n ; t=1,2, \ldots, T .
\end{array}
$$

Equation (11) is the transformation equation and is stochastic. We now have the following propositions.

Proposition 1. If $\gamma=0$ or $\alpha=1$, then

$$
S_{t}^{j}=S_{t-1}^{j} \text {. }
$$

This proposition tells us that the transformation equation (11) is deterministic if $\gamma=0$ or $\alpha=1$. It implies that all the goods distributed from the factories to stores get to their various destination successfully without shortages.

Proposition 2. Suppose that there is no recovery of any of the goods lost in transit and $\gamma>0$; then $\alpha$ must be zero and (11) becomes

$$
\begin{array}{r}
S_{t}^{j}=S_{t-1}^{j}+\gamma \sum_{i=1}^{m} \sum_{p=1}^{q} P_{i, j} x_{p, t}^{\pi_{t}^{j}}\left(S^{f_{i}}\right), \\
j=1,2, \ldots, n ; t=1,2, \ldots, T .
\end{array}
$$

The optimal policy can be found by computing the value functions through the optimality equation

$$
\begin{aligned}
Z_{t}^{\pi_{t}^{j}}\left(S_{t}^{j}\right)= & \min _{x_{t}} \sum_{i=1}^{m} \sum_{p=1}^{q} \Phi_{t}^{i, j}\left(x_{p, t}^{\pi_{t}^{j}}\left(S_{t-1}^{i}\right)\right) \\
& +\beta E\left(Z_{t+1}^{j}\left(S_{t+1}^{j}\right)\right) \mid S_{t}^{j}=s_{t}, \\
& i=1,2, \ldots, m ; t=1,2, \ldots, T,
\end{aligned}
$$

subject to the constraints (5).

Equation (14) can be rewritten as follows:

$$
Z_{t}^{\pi_{t}^{j}}\left(S_{t}^{j}\right)=\min _{x_{t}} \sum_{i=1}^{m} \sum_{p=1}^{q} \Phi_{t}^{i, j}\left(x_{p, t}^{\pi_{t}^{j}}\left(S_{t-1}^{j}\right)\right)+\beta E_{t}\left(Z_{t-1}^{j}\left(S_{t}^{j}\right)\right),
$$

subject to

$$
\begin{gathered}
\sum_{i=1}^{m} \sum_{p=1}^{q} x_{p, t}^{\pi_{t}^{j}}\left(S^{f_{i}}\right) \geq \sum_{j=1}^{n} \sum_{p=1}^{q} x_{p, t}^{\pi_{t}^{j}}\left(S^{s_{j}}\right), \\
x_{p, t}^{\pi_{t}^{j}}\left(S^{f_{i}}\right), x_{p, t}^{\pi_{t}^{j}}\left(S^{s_{j}}\right) \in X, t \in[0, T], p=1,2, \ldots, q . \\
\sum_{p=1}^{q} x_{p, t}^{\pi_{t}^{j}}\left(S^{f_{i}}\right) \geq \sum_{p=1}^{q} x_{p, t}^{\pi_{t}^{j}}\left(S^{s_{j}}\right), \\
x_{p, t}^{\pi_{t}^{j}}\left(S^{f_{i}}\right), x_{p, t}^{\pi_{t}^{j}}\left(S^{s_{j}}\right) \in X, t \in[0, T], p=1,2, \ldots, q .
\end{gathered}
$$

Equivalently,

$$
\begin{gathered}
\sum_{i=1}^{m} \sum_{p=1}^{q} x_{p, t}^{\pi_{t}^{j}}\left(S^{f_{i}}\right) \\
=\sum_{j=1}^{n} \sum_{p=1}^{q} x_{p, t}^{s_{j}}+\delta_{t}, \quad x^{f}, x^{s} \in X, t \in T, \\
i=1,2, \ldots, m ; j=1,2, \ldots, n, \\
x_{p, t}^{f}, x_{p, t}^{s} \geq 0, p=1, \ldots, m . \\
\sum_{i=1}^{m} x_{p, t}^{\pi_{t}^{j}}\left(S^{f_{i}}\right)=\sum_{j=1}^{n} x_{p, t}^{s_{j}}+\delta_{t}^{i, j}, \quad x^{f}, x^{s} \in X, t \in T, \\
i=1,2, \ldots, m ; j=1,2, \ldots, n, \\
x_{p, t}^{f}, x_{p, t}^{s} \geq 0, p=1, \ldots, m,
\end{gathered}
$$

where $\delta_{t}^{i, j} \in \mathbb{R}^{m}$ is the quantity of products that is lost in transit from factory $i$ to store $j$ at period $t$ and $\delta_{t}=\sum_{i=1}^{m} \delta_{t}^{i, j} \epsilon$ $\mathbb{R}$. If $\delta_{t}^{i, j}$ is a zero vector, it implies that all the quantities of products that left the factories get to the stores successfully without damages or shortage. It can be shown that (6) is equal to (14) (see Powell, [22]). We may use (6) and (14) interchangeably. We now find the best control policy, $\mu$, which minimizes our problem; that is, we search for

$$
Z_{t}^{*}\left(S_{t}\right)=\min _{\pi_{t}^{j} \in \mu} Z_{t}^{\pi_{t}^{j}}\left(S_{t}\right), \quad t=1,2, \ldots, T ; S_{t}=S_{t}^{j}
$$

We do that by solving the optimality equation

$$
\begin{aligned}
& Z_{t}^{\pi_{t}^{j}}\left(S_{t}^{j}\right) \\
& \quad=\min _{x_{t}^{f}, x_{t}^{s} \in X} \sum_{i=1}^{m} \sum_{p=1}^{q} \Phi_{t}^{i, j}\left(x_{p, t}^{\pi_{t}^{j}}\left(S_{t-1}^{j}\right)\right)+\beta E_{t}\left(Z_{t-1}^{\pi_{t}^{j}}\left(S_{t}^{j}\right)\right),
\end{aligned}
$$


subject to

$$
\begin{array}{r}
\sum_{p=1}^{q} x_{p, t}^{\pi_{t}^{j}}\left(S^{f_{i}}\right)=\sum_{p=1}^{q} x_{p, t}^{\pi_{t}^{j}}\left(S^{s_{j}}\right) \\
+\delta_{t}^{i, j}, x_{p, t}^{\pi_{t}^{j}}\left(S^{f_{i}}\right), \quad x_{p, t}^{\pi_{t}^{j}}\left(S^{s_{j}}\right) \in X, t \in T, \\
i=1,2, \ldots, m, \quad j=1,2, \ldots, n, \\
\quad x_{p, t}^{f}, x_{p, t}^{s} \geq 0, p=1, \ldots, m .
\end{array}
$$

Let $\Phi\left(S_{t}^{f}, \pi_{t}^{j}, S_{t}^{s}\right)=\sum_{i=1}^{m} \sum_{p=1}^{q} \Phi_{t}^{i, j}\left(x_{p, t}^{\pi_{t}^{j}}\left(S_{t-1}^{j}\right)\right)$ be the expected cost per stage. Using the mapping $\Upsilon: Z \rightarrow \mathbb{R}^{m}$ and $\Upsilon_{\mu}$ : $Z^{\mu} \rightarrow \mathbb{R}^{m}$, we have

$$
\begin{aligned}
(\Upsilon Z)\left(S_{t}^{j}\right)= & \min _{\pi \in \mu\left(S_{t}\right)} \Phi\left(S_{t}^{f}, \pi_{t}^{j}, S_{t}^{s}\right) \\
& +\beta E_{t}\left(Z\left(S_{t}^{j}\right)\right), \quad j=1,2, \ldots, n, \\
\left(\Upsilon_{\mu} Z\right)\left(S_{t}^{j}\right)= & \min _{\pi \in \mu\left(S_{t}\right)} \Phi\left(S_{t}^{f}, \pi_{t}^{j}, S_{t}^{s}\right) \\
& +\beta E_{t}\left(Z^{\mu}\left(S_{t}^{j}\right)\right), \quad j=1,2, \ldots, n .
\end{aligned}
$$

Theorem 3. Consider the following.

(i) Let $B(S)$ be the set of all bounded real-valued functions $Z: S \rightarrow \mathbb{R}^{m}$. The mapping $\Upsilon: B(S) \rightarrow B(S)$ is a contraction.

(ii) The operator $\Upsilon$ has a unique fixed point (given $Z^{*}$ ).

(iii) For any $Z, \Upsilon^{\infty} Z=Z^{*}$.

(iv) For any $Z$, if $\Upsilon Z \leq Z$, then $\Upsilon^{t} Z \geq Z^{*}, \forall t=\{1,2, \ldots\}$.

Proof. (See [22]).

We now make the following assumptions.

Assumption 4. Consider the following.

(i) $\left\|E\left(\sum_{i=1}^{m} \sum_{p=1}^{q} \Phi_{t}^{i, j}\left(x_{p, t}^{\pi_{t}^{j}}\left(S_{t}^{j}\right)\right)\right)\right\| \leq \Lambda_{j}, \quad j=1,2, \ldots, n$.

(ii) The discount factor $0<\beta<1$.

Theorem 5. Let the bounded optimal cost function $Z: S \rightarrow$ $\mathbb{R}^{m}$ be $m$-dimensional vectors. Then $Z$ satisfies

$$
Z^{*}\left(S_{0}^{j}\right)=\lim _{T \rightarrow \infty}\left(\Upsilon^{T} Z\right)\left(S_{0}^{j}\right), \quad \forall S_{0}^{j} \in S .
$$

Proof. Let $y$ be a positive integer, $S_{0}^{j} \in S$ and policy $\mu=$ $\left\{\pi^{1}, \pi^{2}, \ldots, \pi^{n}\right\}$; we can decompose the return

$$
Z^{\mu}\left(S_{0}^{j}\right)=\lim _{T \rightarrow \infty} E\left\{\sum_{t=0}^{T-1} \sum_{i=1}^{m} \sum_{p=1}^{q} \beta^{t} \Phi_{t}^{i, j}\left(x_{p, t}^{\pi_{t}^{j}}\left(S_{t}^{j}\right)\right)\right\}
$$

into the portion received over the first $y$ stages and over the remaining stages:

$$
\begin{aligned}
Z^{\mu}\left(S_{0}^{j}\right)= & \lim _{T \rightarrow \infty} E\left\{\sum_{t=0}^{y-1} \sum_{i=1}^{m} \sum_{p=1}^{q} \beta^{t} \Phi_{t}^{i, j}\left(x_{p, t}^{\pi_{t}^{j}}\left(S_{t}^{j}\right)\right)\right\} \\
& +\lim _{T \rightarrow \infty} E\left\{\sum_{t=y}^{T-1} \sum_{i=1}^{m} \sum_{p=1}^{q} \beta^{t} \Phi_{t}^{i, j}\left(x_{p, t}^{\pi_{t}^{j}}\left(S_{t}^{j}\right)\right)\right\} .
\end{aligned}
$$

But

$$
\begin{gathered}
\left\|\lim _{T \rightarrow \infty} E\left\{\sum_{t=y}^{T-1} \sum_{i=1}^{m} \sum_{p=1}^{q} \beta^{t} \Phi_{t}^{i, j}\left(x_{p, t}^{\pi_{t}^{j}}\left(S_{t}^{j}\right)\right)\right\}\right\| \\
\leq \Lambda_{j} \sum_{t=y}^{\infty} \beta^{t}=\frac{\beta^{y} \Lambda_{j}}{1-\beta} .
\end{gathered}
$$

Therefore,

$$
\begin{aligned}
& Z^{\mu}\left(S_{0}^{j}\right) \\
& \quad \leq \lim _{T \rightarrow \infty} E\left\{\sum_{t=0}^{y-1} \sum_{i=1}^{m} \sum_{p=1}^{q} \beta^{t} \Phi_{t}^{i, j}\left(x_{p, t}^{\pi_{t}^{j}}\left(S_{t}^{j}\right)\right)\right\}+\frac{\beta^{y} \Lambda_{j}}{1-\beta} .
\end{aligned}
$$

It therefore follows that

$$
\begin{aligned}
& Z^{\mu}\left(S_{0}^{j}\right)-\frac{\beta^{y} \Lambda_{j}}{1-\beta}-\beta^{y} \inf _{S_{0}^{j} \in S}\left|\Phi\left(S_{0}^{j}\right)\right| \\
& \leq E\left[\beta^{y} \Phi\left(S_{0}^{j}\right)+\sum_{t=0}^{y-1} \sum_{i=1}^{m} \sum_{p=1}^{q} \beta^{t} \Phi_{t}^{i, j}\left(x_{p, t}^{\pi_{t}^{j}}\left(S_{t}^{j}\right)\right)\right] \\
& \leq Z^{\mu}\left(S_{0}^{j}\right)+\frac{\beta^{y} \Lambda_{j}}{1-\beta}+\beta^{y} \inf _{S_{0}^{j} \in S}\left|\Phi\left(S_{0}^{j}\right)\right| .
\end{aligned}
$$

By taking the infimum over $\mu$, we obtain for all $S_{0}^{j}$ and $y$

$$
\begin{gathered}
Z^{\mu}\left(S_{0}^{j}\right)-\frac{\beta^{y} \Lambda_{j}}{1-\beta}-\beta^{y} \inf _{S_{0}^{j} \in S}\left|\Phi\left(S_{0}^{j}\right)\right| \\
\leq\left(\Upsilon^{y} Z\right)\left(S_{0}^{j}\right) \leq Z^{\mu}\left(S_{0}^{j}\right) \\
+\frac{\beta^{y} \Lambda_{j}}{1-\beta}+\beta^{y} \inf _{S_{0}^{j} \in S}\left|\Phi\left(S_{0}^{j}\right)\right| .
\end{gathered}
$$

and by taking the limit as $y \rightarrow \infty$, we obtain

$$
Z^{*}\left(S_{0}^{j}\right) \leq\left(\Upsilon^{y} Z\right)\left(S_{0}^{j}\right) \leq Z^{*}\left(S_{0}^{j}\right) .
$$

Hence,

$$
Z^{*}\left(S_{0}^{j}\right)=\lim _{y \rightarrow \infty}\left(\Upsilon^{y} Z\right)\left(S_{0}^{j}\right), \quad \forall S_{0}^{j} \in S .
$$


This result shows that our optimization problem converges to a fixed point $Z^{*}$ in an infinite horizon. It therefore follows that

$$
\begin{aligned}
Z^{*}\left(S_{0}^{j}\right)=\min _{\substack{\pi_{t}^{j} \\
x_{p, t} \in X}} E\left[\sum_{i=1}^{m} \sum_{p=1}^{q} \Phi_{t}^{i, j}\left(x_{p, t}^{\pi_{t}^{j}}\left(S_{0}^{j}\right)\right)\right. \\
\\
\left.\quad+\beta Z^{*}\left(x_{p, t}^{\pi_{t}^{j}}\left(S_{0}^{j}\right)\right)\right], \quad \forall S_{0}^{j} \in S .
\end{aligned}
$$

\section{Dynamic Programming Principles with Error Bounds}

In this section, the optimality equation with error bounds is considered. We established the minimum and maximum error bounds of our problem. We show that at infinity the minimum and maximum error bounds cancelled out. Let

$$
Z^{\mu}\left(S_{t}^{j}\right)=\Phi_{\mu}\left(S_{t}^{j}, \mu\left(S_{t}^{j}\right)\right)+\sum_{t=1}^{\infty} \beta^{t} E\left(\Phi\left(S_{t}^{j}, \mu\left(S_{t}^{j}\right)\right) \mid S_{0}^{j}\right)
$$

But

$$
\begin{aligned}
& \Lambda_{j}^{\prime} \leq\left\|\Phi\left(S_{t}^{j}, \mu\left(S_{t}^{j}\right)\right)\right\| \leq \Lambda_{j}^{\prime \prime}, \quad j=1,2, \ldots, n, \\
& \left\|\lim _{T \rightarrow \infty} \beta^{t} E \Phi\left(S_{t}^{j}, \mu\left(S_{t}^{j}\right)\right)\right\| \leq \Lambda_{j} \sum_{t=T}^{\infty} \beta^{t}=\frac{\beta^{T} \Lambda_{j}}{1-\beta} .
\end{aligned}
$$

It then follows that

$$
\Phi_{\mu}+\frac{\beta \Lambda_{j}^{\prime}}{1-\beta} \leq Z^{\mu} \leq \Phi_{\mu}+\frac{\beta \Lambda_{j}^{\prime \prime}}{1-\beta},
$$

where $\Lambda_{j}^{\prime}$ and $\Lambda_{j}^{\prime \prime}$ are such that

$$
\begin{aligned}
& \Lambda_{j}^{\prime}=\min _{S_{t}^{j}} \Phi\left(S_{t}^{j}, \mu\left(S_{t}^{j}\right)\right) . \\
& \Lambda_{j}^{\prime \prime}=\max _{S_{t}^{j}} \Phi\left(S_{t}^{j}, \mu\left(S_{t}^{j}\right)\right) .
\end{aligned}
$$

Equation (37) can be improved upon as follows:

$$
\frac{\Lambda_{j}^{\prime}}{1-\beta} \leq \Phi_{\mu}+\frac{\beta \Lambda_{j}^{\prime}}{1-\beta} \leq Z^{\mu} \leq \Phi_{\mu}+\frac{\beta \Lambda_{j}^{\prime \prime}}{1-\beta} \leq \frac{\Lambda_{j}^{\prime \prime}}{1-\beta} .
$$

For a vector $Z$, we compute

$$
\begin{aligned}
& \Upsilon_{\mu} Z=\Phi_{\mu}+\beta P_{\mu} Z, \\
& Z^{\mu}=\Phi_{\mu}+\beta P_{\mu} Z^{\mu} .
\end{aligned}
$$

By subtracting (40) and (41), we have

$$
Z^{\mu}=\Upsilon_{\mu} Z+\beta P_{\mu}\left(Z^{\mu}-Z\right)
$$

$Z^{\mu}$ is the cost vector associated with the control policy $\mu$ and $Z$ is the cost per stage vector. It then follows from (37) that for

$$
\begin{aligned}
& \eta^{\prime}=\min _{S_{t}^{j}}\left(\left(\Upsilon_{\mu} Z\right)\left(S_{t}^{j}\right)-Z\left(S_{t}^{j}\right)\right), \\
& \eta^{\prime \prime}=\max _{S_{t}^{j}}\left(\left(\Upsilon_{\mu} Z\right)\left(S_{t}^{j}\right)-Z\left(S_{t}^{j}\right)\right),
\end{aligned}
$$

we have

$$
\begin{aligned}
& \frac{\eta^{\prime}}{1-\beta} \leq \Upsilon_{\mu} Z-Z+\frac{\beta \eta^{\prime}}{1-\beta} \\
& \quad \leq Z^{\mu}-Z \leq \Upsilon_{\mu} Z-Z+\frac{\beta \eta^{\prime \prime}}{1-\beta} \leq \frac{\eta^{\prime \prime}}{1-\beta} .
\end{aligned}
$$

Equivalently, for the vector $Z$, we have

$$
Z+\frac{\vartheta^{\prime}}{\beta} \leq \Upsilon_{\mu} Z+\vartheta^{\prime} \leq Z^{\mu} \leq \Upsilon_{\mu} Z+\vartheta^{\prime \prime} \leq Z+\frac{\vartheta^{\prime \prime}}{\beta},
$$

where $\vartheta^{\prime}=\beta \eta^{\prime} /(1-\beta)$ and $\vartheta^{\prime \prime}=\beta \eta^{\prime \prime} /(1-\beta)$. Equation (45) can be improved upon as follows. From (40), we have $\eta^{\prime}=\min _{S_{t}^{j}}\left((\Upsilon Z)\left(S_{t}^{j}\right)-Z\left(S_{t}^{j}\right)\right)$. It follows that

$$
Z+\eta^{\prime} \leq \Upsilon Z
$$

From (46), we obtain

$$
\begin{aligned}
\Upsilon\left(\Upsilon Z-\eta^{\prime}\right) & =\min _{\pi_{t}^{j}}\left\|\Phi_{t}^{\pi_{t}^{j}}+\beta P_{\pi_{t}^{j}}\left(\Upsilon Z-\eta^{\prime}\right)\right\| \\
& =\min _{\pi_{t}^{j}}\left\|\Phi_{t}^{\pi_{t}^{j}}+\beta P_{\pi_{t}^{j}} \Upsilon Z\right\|-\beta \eta^{\prime} \\
& =\Upsilon^{2} Z-\beta \eta^{\prime} .
\end{aligned}
$$

Now, combining (46) and (47), we have

$$
Z+(1-\beta) \beta \eta^{\prime} \leq \Upsilon^{2} Z+\beta^{2} \eta^{\prime} \leq \Upsilon^{3} Z .
$$

Substituting (45) into (50), we have

$$
\begin{aligned}
Z & +\left(1+\beta+\beta^{2}\right) \eta^{\prime} \\
& \leq \Upsilon Z+\left(\beta+\beta^{2}\right) \eta^{\prime} \leq \Upsilon^{2} Z+\beta^{2} \eta^{\prime} \leq \Upsilon^{3} Z .
\end{aligned}
$$

After $k$ steps, we have

$$
\begin{aligned}
Z & +\left(\sum_{i=0}^{k} \beta^{i}\right) \eta^{\prime} \leq \Upsilon Z+\left(\sum_{i=1}^{k} \beta^{i}\right) \eta^{\prime} \\
& \leq \Upsilon^{2} Z+\left(\sum_{i=2}^{k} \beta^{i}\right) \eta^{\prime} \leq \cdots \leq \Upsilon^{k+1} Z .
\end{aligned}
$$

As $k \rightarrow \infty$, we have

$$
Z+\frac{\beta \eta^{\prime}}{\beta(1-\beta)} \leq \Upsilon Z+\frac{\beta \eta^{\prime}}{1-\beta} \leq \Upsilon^{2} Z+\frac{\beta^{2} \eta^{\prime}}{1-\beta} \leq \cdots \leq Z^{*} .
$$

Let $\vartheta_{1}^{\prime}=\beta \eta^{\prime} /(1-\beta)$; we have

$$
Z+\frac{\vartheta_{1}^{\prime}}{\beta} \leq \Upsilon Z+\vartheta_{1}^{\prime} \leq \Upsilon^{2} Z+\beta \vartheta_{1}^{\prime} \leq Z^{*} .
$$


Let $Z=\Upsilon^{k+1} Z$ in (54); we have

$$
\Upsilon^{k+1} Z+\vartheta_{k+1}^{\prime} \leq Z^{*}
$$

From (46), we have that $\min _{S_{t}^{j}}\left(\left(\Upsilon^{2} Z\right)\left(S_{t}^{j}\right)-(\Upsilon Z)\left(S_{t}^{j}\right)\right) \geq \beta \eta^{\prime}$ and $\beta 9_{1}^{\prime}=\left(\beta^{2} \eta^{\prime} /(1-\beta)\right) \leq \vartheta_{2}^{\prime}$. Therefore, we have

$$
\Upsilon Z+\vartheta_{1}^{\prime} \leq \Upsilon^{2} Z+\vartheta_{2}^{\prime} \leq Z^{*} .
$$

It implies that

$$
\left(\Upsilon^{k} Z\right)\left(S_{t}^{j}\right)+\vartheta_{k}^{\prime} \leq\left(\Upsilon^{k+1} Z\right)\left(S_{t}^{j}\right)+\vartheta_{k+1}^{\prime} \leq Z^{*}\left(S_{t}^{j}\right) .
$$

Similarly, $\eta^{\prime \prime}=\|\Upsilon Z-Z\|_{\infty}$. We therefore have that

$$
\begin{gathered}
\left(\Upsilon^{k} Z\right)\left(S_{t}^{j}\right)+\vartheta_{k}^{\prime} \leq\left(\Upsilon^{k+1} Z\right)\left(S_{t}^{j}\right)+\vartheta_{k+1}^{\prime} \\
\leq Z^{*}\left(S_{t}^{j}\right) \leq\left(\Upsilon^{k+1} Z\right)\left(S_{t}^{j}\right) \\
\quad+\vartheta_{k+1}^{\prime \prime} \leq\left(\Upsilon^{k} Z\right)\left(S_{t}^{j}\right)+\vartheta_{k}^{\prime \prime} .
\end{gathered}
$$

This is the dynamic programming (value iteration) that is bounded above and below. The value $\left(\Upsilon^{k} Z\right)\left(S_{t}^{j}\right)+\vartheta_{k}^{\prime}$, is the value iteration with minimum error bound and $\left(\Upsilon^{k} Z\right)\left(S_{t}^{j}\right)+$ $\vartheta_{k}^{\prime \prime}$ is the value iteration with maximum error bound. Now taking the limit as $k$ tends to infinity, we have that $\vartheta_{\infty}^{\prime}=\vartheta_{\infty}^{\prime \prime}=$ 0 ; it now implies that

$$
\begin{aligned}
& \lim _{k \rightarrow \infty}\left(\left(\Upsilon^{k} Z\right)\left(S_{t}^{j}\right)+9_{k}^{\prime}\right) \\
& \quad=\lim _{k \rightarrow \infty}\left(\left(\Upsilon^{k} Z\right)\left(S_{t}^{j}\right)+\vartheta_{k}^{\prime \prime}\right)=\left(\Upsilon^{\infty} Z\right)\left(S^{j}\right),
\end{aligned}
$$

and for $\bar{k}<\infty$,

$$
\lim _{k \rightarrow \bar{k}}\left(\left(\Upsilon^{k} Z\right)\left(S_{t}^{j}\right)+\vartheta_{k}^{\prime}\right) \neq \lim _{k \rightarrow \bar{k}}\left(\left(\Upsilon^{k} Z\right)\left(S_{t}^{j}\right)+\vartheta_{k}^{\prime \prime}\right) .
$$

$$
\Phi_{0}^{i j}=\left(\begin{array}{cccccccc}
120.04 & 56.8 & 100 & 75 & 80.5 & 90 & 92 & 99.04 \\
110 & 80.9 & 120 & 60 & 65 & 70 & 75 & 88.9 \\
78.9 & 105 & 64 & 132 & 84.2 & 93 & 95 & 87.09 \\
59.8 & 140 & 92 & 112.8 & 62 & 69 & 75.3 & 78.1 \\
108 & 101 & 85.5 & 72.05 & 76.5 & 66.4 & 65.3 & 99.05 \\
105.6 & 112.6 & 90.1 & 80.4 & 79.5 & 67.5 & 59 & 75.9 \\
112.03 & 109.2 & 80.25 & 80 & 77 & 75.2 & 69.9 & 77.08 \\
113 & 111.01 & 97.8 & 79.9 & 89.5 & 60.8 & 92.02 & 77.09
\end{array}\right) .
$$

The company estimates $\gamma=5 \%$ of the total goods as shortage in transit and $\alpha=70 \%$ of the total expected shortage to be recovered and should be deducted from the total cost of the

\section{Numerical Simulation}

In this section, we present the numerical simulation of the company problem. The state transition diagram of the distribution of goods from factories to stores, under the control policies $\pi^{1}, \pi^{2}, \pi^{3}, \pi^{4}, \pi^{5}, \pi^{6}, \pi^{7}, \pi^{8}$, is given by the transition diagram in Figure 1.

The transition probability matrix of distributing the goods from factory $i$ to store $j$ under the control policies $\pi^{1}, \pi^{2}, \pi^{3}, \pi^{4}, \pi^{5}, \pi^{6}, \pi^{7}, \pi^{8}$, is presented below:

$$
P_{i j}\left(\pi^{j}\right)=\left(\begin{array}{cccccccc}
\frac{7}{40} & \frac{3}{40} & \frac{1}{40} & \frac{1}{5} & \frac{1}{10} & \frac{1}{20} & \frac{1}{4} & \frac{1}{8} \\
\frac{1}{8} & \frac{1}{4} & \frac{1}{20} & \frac{1}{10} & \frac{1}{5} & \frac{1}{40} & \frac{3}{40} & \frac{7}{40} \\
\frac{1}{5} & \frac{7}{40} & \frac{1}{10} & \frac{1}{20} & \frac{1}{4} & \frac{1}{8} & \frac{1}{40} & \frac{3}{40} \\
\frac{1}{10} & \frac{1}{8} & \frac{1}{4} & \frac{3}{40} & \frac{1}{40} & \frac{1}{5} & \frac{7}{40} & \frac{1}{20} \\
\frac{3}{40} & \frac{1}{5} & \frac{7}{40} & \frac{1}{4} & \frac{1}{8} & \frac{1}{10} & \frac{1}{20} & \frac{1}{40} \\
\frac{1}{40} & \frac{1}{10} & \frac{3}{40} & \frac{1}{8} & \frac{1}{20} & \frac{7}{40} & \frac{1}{5} & \frac{1}{4} \\
\frac{1}{4} & \frac{1}{20} & \frac{1}{8} & \frac{1}{40} & \frac{7}{40} & \frac{3}{40} & \frac{1}{10} & \frac{1}{5} \\
\frac{1}{20} & \frac{1}{40} & \frac{1}{5} & \frac{7}{40} & \frac{3}{40} & \frac{1}{4} & \frac{1}{8} & \frac{1}{10}
\end{array}\right) .
$$

The initial estimate of the cost of distributing the goods from factory $i$ to store $j$ in 100000 is given below.

shipment. The cost of the damaged goods is added to the cost of the shipment. MATLAB was used to solve the problem. The results are presented in the tables. 


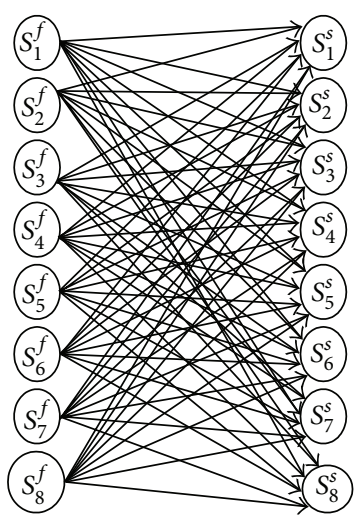

FIGURE 1: Transition diagram for the distribution of goods from factories to stores.

TABLE 1: ODCDG without error bound (EB).

\begin{tabular}{|c|c|c|c|c|c|c|c|c|}
\hline$k$ & $\begin{array}{c}\Upsilon^{k}(Z)\left(S_{1}\right) \times \\
1000000 \\
\text { (naira) }\end{array}$ & $\begin{array}{c}\Upsilon^{k}(Z)\left(S_{2}\right) \times \\
1000000 \\
\text { (naira) }\end{array}$ & $\begin{array}{c}\Upsilon^{k}(Z)\left(S_{3}\right) \times \\
1000000 \\
(\text { naira })\end{array}$ & $\begin{array}{c}\Upsilon^{k}(Z)\left(S_{4}\right) \times \\
1000000 \\
\text { (naira) }\end{array}$ & $\begin{array}{c}Y^{k}(Z)\left(S_{5}\right) \times \\
1000000 \\
\text { (naira) }\end{array}$ & $\begin{array}{c}\Upsilon^{k}(Z)\left(S_{6}\right) \times \\
1000000 \\
\text { (naira) }\end{array}$ & $\begin{array}{c}\Upsilon^{k}(Z)\left(S_{7}\right) \times \\
1000000 \\
\text { (naira) }\end{array}$ & $\begin{array}{c}Y^{k}(Z)\left(S_{8}\right) \times \\
1000000 \\
\text { (naira) }\end{array}$ \\
\hline 0 & 0.0000 & 0.0000 & 0.0000 & 0.0000 & 0.0000 & 0.0000 & 0.0000 & 0.0000 \\
\hline 1 & 5.6232 & 5.9400 & 6.3360 & 5.9202 & 6.4647 & 5.8410 & 6.9201 & 6.0192 \\
\hline 2 & 5.7148 & 6.0326 & 6.4270 & 6.0131 & 6.5563 & 5.9326 & 7.0117 & 6.1120 \\
\hline 3 & 5.7162 & 6.0340 & 6.4283 & 6.0145 & 6.5577 & 5.9340 & 7.0131 & 6.1133 \\
\hline$\infty$ & 5.7162 & 6.0340 & 6.4284 & 6.0145 & 6.5577 & 5.9340 & 7.0131 & 6.1134 \\
\hline
\end{tabular}

ODCDG stands for optimal discounted costs of distributing the goods.

TABLE 2: ODCDG with minimum EB.

\begin{tabular}{|c|c|c|c|c|c|c|c|c|}
\hline$k$ & $\begin{array}{c}\Upsilon^{k}(Z)\left(S_{1}\right)+ \\
\vartheta^{\prime} \times 10^{8} \\
(\text { naira })\end{array}$ & $\begin{array}{c}\Upsilon^{k}(Z)\left(S_{2}\right)+ \\
\vartheta^{\prime} \times 10^{8} \\
\text { (naira) }\end{array}$ & $\begin{array}{c}Y^{k}(Z)\left(S_{3}\right)+ \\
\vartheta^{\prime} \times 10^{8} \\
(\text { naira })\end{array}$ & $\begin{array}{c}\Upsilon^{k}(Z)\left(S_{4}\right)+ \\
9^{\prime} \times 10^{8} \\
(\text { naira })\end{array}$ & $\begin{array}{c}\Upsilon^{k}(Z)\left(S_{5}\right)+ \\
9^{\prime} \times 10^{8} \\
\text { (naira) }\end{array}$ & $\begin{array}{c}\Upsilon^{k}(Z)\left(S_{6}\right)+ \\
\vartheta^{\prime} \times 10^{8} \\
(\text { naira })\end{array}$ & $\begin{array}{c}\Upsilon^{k}(Z)\left(S_{7}\right)+ \\
\vartheta^{\prime} \times 10^{8} \\
(\text { naira })\end{array}$ & $\begin{array}{c}\Upsilon^{k}(Z)\left(S_{8}\right)+ \\
\vartheta^{\prime} \times 10^{8} \\
(\text { naira })\end{array}$ \\
\hline 0 & - & - & - & - & - & - & - & - \\
\hline 1 & 5.6232 & 5.6264 & 5.6303 & 5.6262 & 5.6316 & 5.6254 & 5.6362 & 5.6272 \\
\hline 2 & 0.1472 & 0.1504 & 0.1543 & 0.1502 & 0.1556 & 0.1494 & 0.1602 & 0.1512 \\
\hline 3 & 0.0585 & 0.0617 & 0.0656 & 0.0602 & 0.0669 & 0.0607 & 0.0715 & 0.0625 \\
\hline 4 & 0.0572 & 0.0604 & 0.0643 & 0.0601 & 0.0656 & 0.0594 & 0.0702 & 0.0612 \\
\hline$\infty$ & 0.0572 & 0.0603 & 0.0643 & 0.0601 & 0.0656 & 0.0593 & 0.0701 & 0.0611 \\
\hline
\end{tabular}

The optimal discounted costs of distributing the goods from factories to stores with shortages are presented in Tables 1 to 7 and Figures 2 and 3, while optimal discounted costs of distributing the goods from factories to stores without shortages are presented in Tables 8 to 10 and Figure 5. Table 1 presents the minimum costs of distributing the goods from various factories to all the stores. Here, error bound is not considered. It was obtained that the minimum costs of distributing the goods from factory 1 to all the eight stores are $5,716,200$ naira. The minimum costs of distributing the goods from factory 2 to the eight stores are obtained as $6,034,000$ naira, from factory 3 to the eight stores are $6,428,400$ naira, and so on. It was observed that the costs of distributing the goods from factory 7 to all the stores yield the highest amount. This may be due to longer distance, nature of the roads, or more shortage costs.
Table 2 presents the optimal costs of distributing goods with minimum error bound from the factories to stores and Table 3 presents the optimal costs of distributing the goods with maximum error bound from the factories to stores. It was observed that the minimum costs of distributing the goods with minimum and maximum error bounds coincide only at infinity.

Table 4 shows the optimal costs of distributing the goods without error bound and where there is no recovery of some damaged goods (i.e., when $\alpha=0$ ). It is observed from Tables 1 and 4 that the costs of distributing the goods will increase when the value of $\alpha$ decreases and vice versa. This is an expected result. Tables 5 and 6 show the optimal costs of distributing the goods with minimum and maximum error bounds, respectively, and where there is no recovery of some damaged goods. 
TABLE 3: ODCDG with maximum EB.

\begin{tabular}{|c|c|c|c|c|c|c|c|c|}
\hline$k$ & $\begin{array}{c}\Upsilon^{k}(Z)\left(S_{1}\right)+ \\
9^{\prime \prime} \times 10^{8} \text { (naira) }\end{array}$ & $\begin{array}{c}\Upsilon^{k}(Z)\left(S_{2}\right)+ \\
9^{\prime \prime} \times 10^{8} \text { (naira) }\end{array}$ & $\begin{array}{c}\Upsilon^{k}(Z)\left(S_{3}\right)+ \\
9^{\prime \prime} \times 10^{8} \text { (naira) }\end{array}$ & $\begin{array}{c}\Upsilon^{k}(Z)\left(S_{4}\right)+ \\
9^{\prime \prime} \times 10^{8} \text { (naira) }\end{array}$ & $\begin{array}{c}Y^{k}(Z)\left(S_{5}\right)+ \\
9^{\prime \prime} \times 10^{8} \text { (naira) }\end{array}$ & $\begin{array}{c}\Upsilon^{k}(Z)\left(S_{6}\right)+ \\
9^{\prime \prime} \times 10^{8}(\text { naira })\end{array}$ & $\begin{array}{c}\Upsilon^{k}(Z)\left(S_{7}\right)+ \\
9^{\prime \prime} \times 10^{8}(\text { naira })\end{array}$ & $\begin{array}{c}\Upsilon^{k}(Z)\left(S_{8}\right)+ \\
9^{\prime \prime} \times 10^{8}(\text { naira })\end{array}$ \\
\hline 0 & - & - & - & - & - & - & - & - \\
\hline 1 & 6.9071 & 6.9103 & 6.9143 & 6.9101 & 6.9155 & 6.9093 & 6.9201 & 6.9111 \\
\hline 2 & 0.1491 & 0.1523 & 0.1562 & 0.1521 & 0.1575 & 0.1513 & 0.1621 & 0.1531 \\
\hline 3 & 0.0585 & 0.0617 & 0.0657 & 0.0615 & 0.0669 & 0.0607 & 0.0715 & 0.0625 \\
\hline 4 & 0.0572 & 0.0604 & 0.0643 & 0.0602 & 0.0656 & 0.0594 & 0.0702 & 0.0612 \\
\hline$\infty$ & 0.0572 & 0.0603 & 0.0643 & 0.0601 & 0.0656 & 0.0593 & 0.0701 & 0.0611 \\
\hline
\end{tabular}

TABLE 4: ODCDG where there is no recovery; that is, when $\alpha=0$.

\begin{tabular}{ccccccccc}
\hline$k$ & $\begin{array}{c}\Upsilon^{k}(Z)\left(S_{1}\right) \times \\
1000000 \\
\text { (naira) }\end{array}$ & $\begin{array}{c}\Upsilon^{k}(Z)\left(S_{2}\right) \times \\
1000000 \\
\text { (naira) }\end{array}$ & $\begin{array}{c}\Upsilon^{k}(Z)\left(S_{3}\right) \times \\
\text { (naira) }\end{array}$ & $\begin{array}{c}\Upsilon^{k}(Z)\left(S_{4}\right) \times \\
\text { (naira) }\end{array}$ & $\begin{array}{c}\Upsilon^{k}(Z)\left(S_{5}\right) \times \\
1000000 \\
\text { (naira) }\end{array}$ & $\begin{array}{c}\Upsilon^{k}(Z)\left(S_{6}\right) \times \\
1000000 \\
\text { (naira) }\end{array}$ & $\begin{array}{c}\Upsilon^{k}(Z)\left(S_{7}\right) \times \\
1000000 \\
\text { (naira) }\end{array}$ & $\begin{array}{c}\Upsilon^{k}(Z)\left(S_{8}\right) \times \\
1000000 \\
\text { (naira) }\end{array}$ \\
\hline 0 & 0.0000 & 0.0000 & 0.0000 & 0.0000 & 0.0000 & 0.0000 & 0.0000 \\
1 & 5.6232 & 5.9400 & 6.3360 & 5.9202 & 6.4647 & 5.8410 & 6.9201 \\
2 & 5.9286 & 6.2487 & 6.6392 & 6.2298 & 6.7701 & 6.1464 & 7.2255 \\
3 & 5.9440 & 6.2640 & 6.6545 & 6.2452 & 6.7854 & 6.1617 & 7.2408 & 6.0192 \\
4 & 5.9447 & 6.2648 & 6.6553 & 6.2460 & 6.7862 & 6.1625 & 7.2416 & 6.3438 \\
$\infty$ & 5.9448 & 6.2648 & 6.6553 & 6.2460 & 6.7862 & 6.1625 & 7.2416 \\
\hline
\end{tabular}

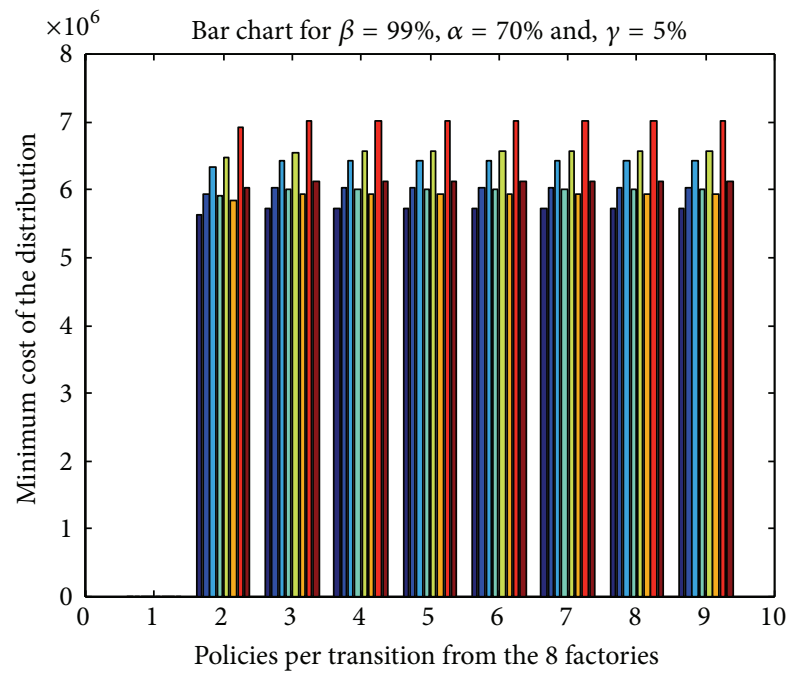

$\begin{array}{ll}\square \text { Policy } 1 & \square \text { Policy } 5 \\ \square \text { Policy 2 } & \square \text { Policy 6 } \\ \square \text { Policy 3 } & \square \text { Policy 7 } \\ \square \text { Policy } 4 & \square \text { Policy 8 }\end{array}$

FIGURE 2: The bar chart representing the discounted minimum costs of the distribution with 8 different policies for $\beta=99 \%, \alpha=70 \%$, and $\gamma=5 \%$.

The additional costs incurred and the corresponding percentage increase in the additional costs incurred when $\alpha=0$ are shown in Table 7 .

In Table 7, it was found that the costs of recovered goods in transit from factory 1 to the stores are 228,600 naira with

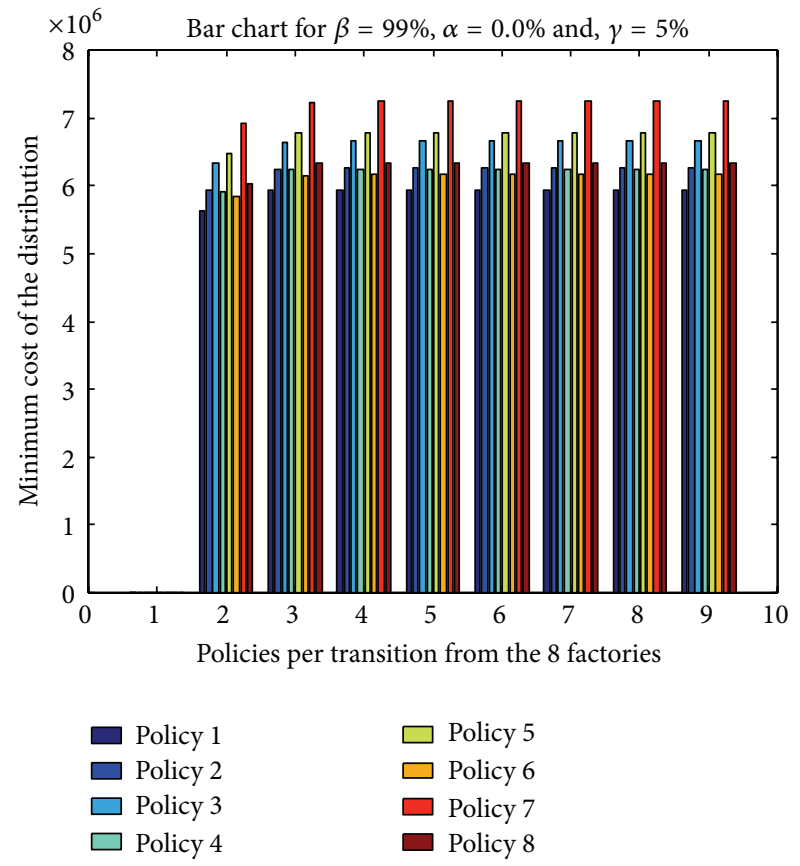

FIGURE 3: The bar chart representing the discounted minimum cost of the distribution with 8 different policies for $\beta=99 \%, \alpha=0.0 \%$, and $\gamma=5 \%$. This occurs when there is no recovery of the goods.

$3.85 \%$ when there is no $\mathrm{EB}$ and 220000 naira with $3.70 \%$ when there is EB from factory 2 to the stores 230,800 with $3.68 \%$ when there is no EB, and 240,000 with $3.83 \%$ when there is $\mathrm{EB}$ and so on. 
TABLE 5: ODCDG with minimum EB and where there is no recovery.

\begin{tabular}{|c|c|c|c|c|c|c|c|c|}
\hline$k$ & $\begin{array}{c}\Upsilon^{k}(Z)\left(S_{1}\right)+ \\
\vartheta^{\prime} \times 10^{8} \\
(\text { naira })\end{array}$ & $\begin{array}{c}\Upsilon^{k}(Z)\left(S_{2}\right)+ \\
9^{\prime} \times 10^{8} \\
(\text { naira })\end{array}$ & $\begin{array}{c}\Upsilon^{k}(Z)\left(S_{3}\right)+ \\
\vartheta^{\prime} \times 10^{8} \\
(\text { naira })\end{array}$ & $\begin{array}{c}\Upsilon^{k}(Z)\left(S_{4}\right)+ \\
\vartheta^{\prime} \times 10^{8} \\
(\text { naira })\end{array}$ & $\begin{array}{c}\Upsilon^{k}(Z)\left(S_{5}\right)+ \\
9^{\prime} \times 10^{8} \\
\text { (naira) }\end{array}$ & $\begin{array}{c}\Upsilon^{k}(Z)\left(S_{6}\right)+ \\
\vartheta^{\prime} \times 10^{8} \\
\text { (naira) }\end{array}$ & $\begin{array}{c}\Upsilon^{k}(Z)\left(S_{7}\right)+ \\
\vartheta^{\prime} \times 10^{8} \\
\text { (naira) }\end{array}$ & $\begin{array}{c}\Upsilon^{k}(Z)\left(S_{8}\right)+ \\
\mathcal{G}^{\prime} \times 10^{8} \\
\quad \text { (naira) }\end{array}$ \\
\hline 0 & - & - & - & - & - & - & - & - \\
\hline 1 & 5.6232 & 5.6264 & 5.6303 & 5.6262 & 5.6316 & 5.6254 & 5.6362 & 5.6272 \\
\hline 2 & 0.3595 & 0.3627 & 0.3666 & 0.3625 & 0.3679 & 0.3617 & 0.3724 & 0.3635 \\
\hline 3 & 0.0746 & 0.0778 & 0.0817 & 0.0776 & 0.0830 & 0.0768 & 0.0875 & 0.0786 \\
\hline 4 & 0.0602 & 0.0634 & 0.0673 & 0.0632 & 0.0686 & 0.0624 & 0.0732 & 0.0642 \\
\hline 5 & 0.0595 & 0.0627 & 0.0666 & 0.0625 & 0.0679 & 0.0617 & 0.0725 & 0.0635 \\
\hline$\infty$ & 0.0594 & 0.0627 & 0.0666 & 0.0625 & 0.0679 & 0.0616 & 0.0724 & 0.0634 \\
\hline
\end{tabular}

TABLE 6: ODCDG with maximum EB where there is no recovery.

\begin{tabular}{|c|c|c|c|c|c|c|c|c|}
\hline$k$ & $\begin{array}{c}\Upsilon^{k}(Z)\left(S_{1}\right)+ \\
\vartheta^{\prime \prime} \times 10^{8} \\
\text { (naira) }\end{array}$ & $\begin{array}{c}\Upsilon^{k}(Z)\left(S_{2}\right)+ \\
\vartheta^{\prime \prime} \times 10^{8} \\
\text { (naira) }\end{array}$ & $\begin{array}{c}\Upsilon^{k}(Z)\left(S_{3}\right)+ \\
9^{\prime \prime} \times 10^{8} \\
(\text { naira })\end{array}$ & $\begin{array}{c}\Upsilon^{k}(Z)\left(S_{4}\right)+ \\
9^{\prime \prime} \times 10^{8} \\
(\text { naira })\end{array}$ & $\begin{array}{c}\Upsilon^{k}(Z)\left(S_{5}\right)+ \\
9^{\prime \prime} \times 10^{8} \\
(\text { naira })\end{array}$ & $\begin{array}{c}\Upsilon^{k}(Z)\left(S_{6}\right)+ \\
9^{\prime \prime} \times 10^{8} \\
(\text { naira })\end{array}$ & $\begin{array}{c}\Upsilon^{k}(Z)\left(S_{7}\right)+ \\
9^{\prime \prime} \times 10^{8} \\
\text { (naira) }\end{array}$ & $\begin{array}{c}\Upsilon^{k}(Z)\left(S_{8}\right)+ \\
9^{\prime \prime} \times 10^{8} \\
(\text { naira })\end{array}$ \\
\hline 0 & - & - & - & - & - & - & - & - \\
\hline 1 & 6.9071 & 6.9103 & 6.9143 & 6.9101 & 6.9155 & 6.9093 & 6.9201 & 6.9111 \\
\hline 2 & 0.3658 & 0.3690 & 0.3729 & 0.3688 & 0.3743 & 0.3680 & 0.3788 & 0.3698 \\
\hline 3 & 0.0746 & 0.0778 & 0.0818 & 0.0777 & 0.0831 & 0.0768 & 0.0876 & 0.0786 \\
\hline 4 & 0.0602 & 0.0634 & 0.0673 & 0.0632 & 0.0686 & 0.0624 & 0.0732 & 0.0642 \\
\hline 5 & 0.0595 & 0.0627 & 0.0666 & 0.0625 & 0.0679 & 0.0617 & 0.0725 & 0.0635 \\
\hline$\infty$ & 0.0594 & 0.0627 & 0.0666 & 0.0625 & 0.0679 & 0.0616 & 0.0724 & 0.0634 \\
\hline
\end{tabular}

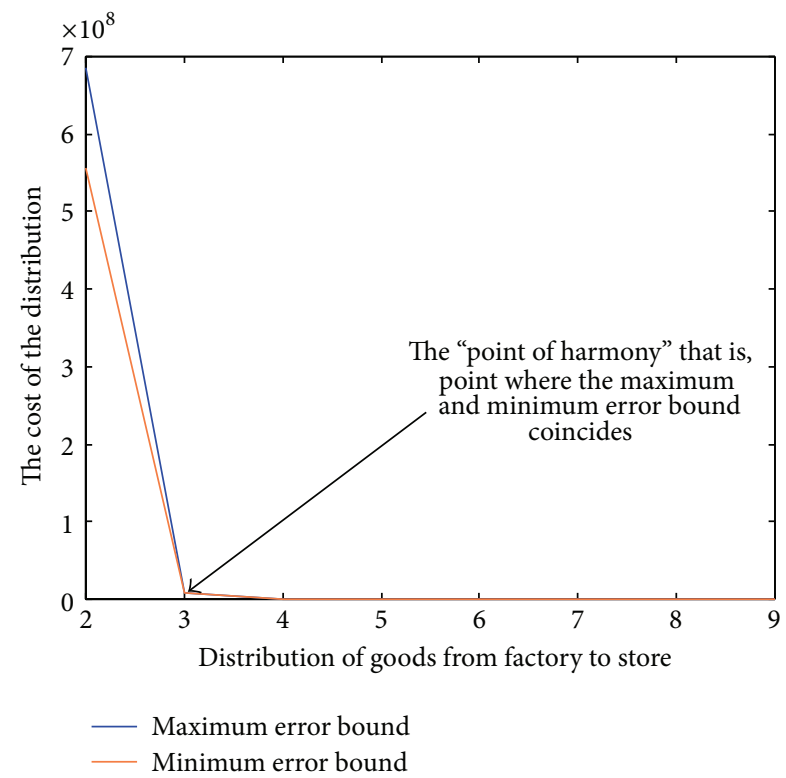

FIGURE 4: The maximum and minimum error bound showing the point of their intersection "point of harmony" at $\beta=99 \%, \alpha=70 \%$, and $\gamma=5 \%$.

Figure 2 shows the bar chart representing the minimum costs of distributing the goods from factories to stores by setting $\beta=99 \%, \alpha=70 \%$, and $\gamma=5 \%$. It was observed from

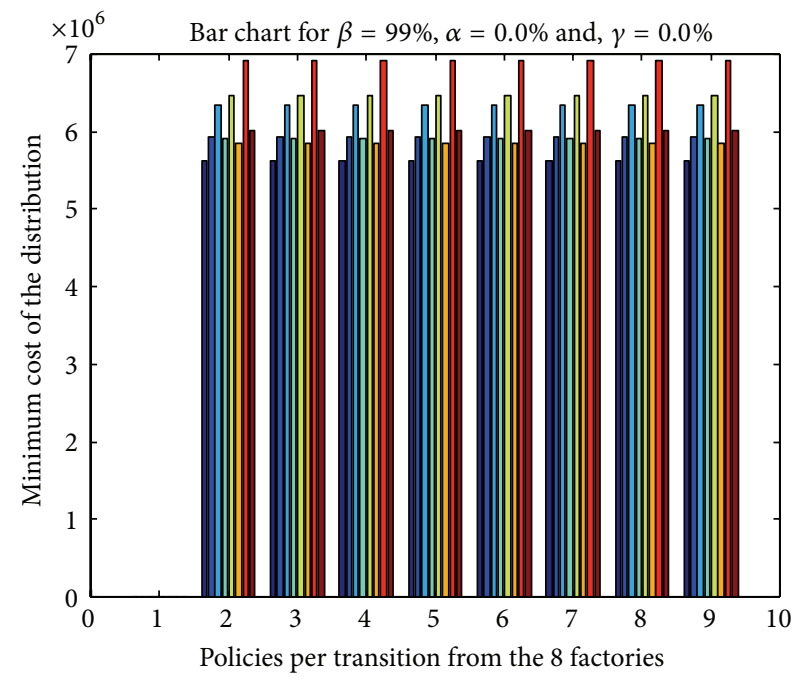

FIGURE 5: The bar chart representing the discounted minimum cost of the distribution with 8 different policies for $\beta=99 \%, \alpha=0.0 \%$, and $\gamma=0.0 \%$. This is when the goods get to their various stores successfully without shortages.

Figure 2 that optimum costs can be attained under policy 1. Hence, the optimum policy for the operation is $\pi^{1}$. We therefore deduce from Figure 2 that $\pi^{1}<\pi^{6}<\pi^{4}<\pi^{2}<$ $\pi^{8}<\pi^{3}<\pi^{5}<\pi^{7}$. Figure 3 shows the bar chart representing the minimum costs of distributing the goods from factories to 
TABLE 7: Optimal discounted costs of recovered goods.

\begin{tabular}{ccccc}
\hline$m$ & $\begin{array}{c}\text { Optimal costs of } \\
\text { recovered goods } \\
\text { without EB (Naira) }\end{array}$ & $\begin{array}{c}\text { \% Optimal costs of } \\
\text { recovered goods } \\
\text { without EB }\end{array}$ & $\begin{array}{c}\text { Optimal costs of } \\
\text { recovered goods with } \\
\text { EB (Naira) }\end{array}$ & $\begin{array}{c}\text { \% Optimal costs of } \\
\text { recovered goods with } \\
\text { EB }\end{array}$ \\
\hline 1 & 228600 & 3.85 & 220000 & 3.70 \\
2 & 230800 & 3.68 & 240000 & 3.83 \\
3 & 226900 & 3.41 & 230000 & 3.45 \\
4 & 231500 & 3.71 & 240000 & 3.84 \\
5 & 228500 & 3.37 & 230000 & 3.39 \\
6 & 228500 & 3.71 & 230000 & 3.73 \\
7 & 228500 & 3.16 & 230000 & 3.18 \\
8 & 231200 & 3.64 & 230000 & 3.63 \\
\hline
\end{tabular}

TABLE 8: ODCDG without shortages.

\begin{tabular}{|c|c|c|c|c|c|c|c|c|}
\hline$k$ & $\begin{array}{c}\Upsilon^{k}(Z)\left(S_{1}\right) \times \\
1000000 \\
\text { (naira) }\end{array}$ & $\begin{array}{c}\Upsilon^{k}(Z)\left(S_{2}\right) \times \\
1000000 \\
\text { (naira) }\end{array}$ & $\begin{array}{c}\Upsilon^{k}(Z)\left(S_{3}\right) \times \\
1000000 \\
\text { (naira) }\end{array}$ & $\begin{array}{c}\Upsilon^{k}(Z)\left(S_{4}\right) \times \\
1000000 \\
\text { (naira) }\end{array}$ & $\begin{array}{c}\Upsilon^{k}(Z)\left(S_{5}\right) \times \\
1000000 \\
\text { (naira) }\end{array}$ & $\begin{array}{c}\Upsilon^{k}(Z)\left(S_{6}\right) \times \\
1000000 \\
\text { (naira) }\end{array}$ & $\begin{array}{c}\Upsilon^{k}(Z)\left(S_{7}\right) \times \\
1000000 \\
\text { (naira) }\end{array}$ & $\begin{array}{c}Y^{k}(Z)\left(S_{8}\right) \times \\
1000000 \\
\text { (naira) }\end{array}$ \\
\hline 0 & 0.0000 & 0.0000 & 0.0000 & 0.0000 & 0.0000 & 0.0000 & 0.0000 & 0.0000 \\
\hline 1 & 5.6232 & 5.9400 & 6.3360 & 5.9202 & 6.4647 & 5.8410 & 6.9201 & 6.0192 \\
\hline$\infty$ & 5.6232 & 5.9400 & 6.3360 & 5.9202 & 6.4647 & 5.8410 & 6.9201 & 6.0192 \\
\hline
\end{tabular}

TABLE 9: ODCDG without shortage with minimum EB.

\begin{tabular}{|c|c|c|c|c|c|c|c|c|}
\hline$k$ & $\begin{array}{c}\Upsilon^{k}(Z)\left(S_{1}\right)+ \\
9^{\prime} \times 10^{8}(\text { naira })\end{array}$ & $\begin{array}{c}\Upsilon^{k}(Z)\left(S_{2}\right)+ \\
9^{\prime} \times 10^{8}(\text { naira })\end{array}$ & $\begin{array}{c}\Upsilon^{k}(Z)\left(S_{3}\right)+ \\
\vartheta^{\prime} \times 10^{8} \text { (naira) }\end{array}$ & $\begin{array}{c}\Upsilon^{k}(Z)\left(S_{4}\right)+ \\
9^{\prime} \times 10^{8} \text { (naira) }\end{array}$ & $\begin{array}{c}\Upsilon^{k}(Z)\left(S_{5}\right)+ \\
9^{\prime} \times 10^{8} \text { (naira) }\end{array}$ & $\begin{array}{c}\Upsilon^{k}(Z)\left(S_{6}\right)+ \\
9^{\prime} \times 10^{8} \text { (naira) }\end{array}$ & $\begin{array}{c}\Upsilon^{k}(Z)\left(S_{7}\right)+ \\
9^{\prime} \times 10^{8} \text { (naira) }\end{array}$ & $\begin{array}{c}\Upsilon^{k}(Z)\left(S_{8}\right)+ \\
\mathcal{\vartheta}^{\prime} \times 10^{8} \\
\quad(\text { naira })\end{array}$ \\
\hline 0 & - & - & - & - & - & - & - & - \\
\hline 1 & 5.6232 & 5.6264 & 5.6303 & 5.6262 & 5.6316 & 5.6254 & 5.6362 & 5.6272 \\
\hline$\infty$ & 0.0562 & 0.0594 & 0.0634 & 0.0592 & 0.0646 & 0.0584 & 0.0692 & 0.0602 \\
\hline
\end{tabular}

TABLE 10: ODCDG without shortage with maximum EB.

\begin{tabular}{|c|c|c|c|c|c|c|c|c|}
\hline$k$ & $\begin{array}{c}\Upsilon^{k}(Z)\left(S_{1}\right)+ \\
9^{\prime \prime} \times 10^{8} \\
\text { (naira) }\end{array}$ & $\begin{array}{c}\Upsilon^{k}(Z)\left(S_{2}\right)+ \\
\mathcal{9}^{\prime \prime} \times 10^{8} \\
\text { (naira) }\end{array}$ & $\begin{array}{c}\Upsilon^{k}(Z)\left(S_{3}\right)+ \\
9^{\prime \prime} \times 10^{8} \\
(\text { naira })\end{array}$ & $\begin{array}{c}\Upsilon^{k}(Z)\left(S_{4}\right)+ \\
9^{\prime \prime} \times 10^{8} \\
(\text { naira })\end{array}$ & $\begin{array}{c}\Upsilon^{k}(Z)\left(S_{5}\right)+ \\
\vartheta^{\prime \prime} \times 10^{8} \\
\text { (naira) }\end{array}$ & $\begin{array}{c}\Upsilon^{k}(Z)\left(S_{6}\right)+ \\
9^{\prime \prime} \times 10^{8} \\
\text { (naira) }\end{array}$ & $\begin{array}{c}Y^{k}(Z)\left(S_{7}\right)+ \\
\mathcal{9}^{\prime \prime} \times 10^{8} \\
\text { (naira) }\end{array}$ & $\begin{array}{c}\Upsilon^{k}(Z)\left(S_{8}\right)+ \\
\vartheta^{\prime \prime} \times 10^{8} \\
\text { (naira) }\end{array}$ \\
\hline 0 & - & - & - & - & - & - & - & - \\
\hline 1 & 6.9071 & 6.9103 & 6.9143 & 6.9101 & 6.9155 & 6.9093 & 6.9201 & 6.9111 \\
\hline$\infty$ & 0.0562 & 0.0594 & 0.0634 & 0.0592 & 0.0646 & 0.0584 & 0.0692 & 0.0602 \\
\hline
\end{tabular}

stores by setting $\beta=99 \%, \alpha=0.0 \%$, and $\gamma=5 \%$. This is when there are no goods recovered from the expected lost ones in transit. We also found that policy 1 yields the optimum costs. Figure 4 shows the relationship between the minimum and maximum error bound. It was observed that at infinity, the minimum and maximum error bounds coincide. We referred to this point as the point of harmony. Comparing the optimal discounted costs of the distribution of goods in Tables 1, 8, 2, 9, 3, and 10 and Figures 2 and 5, we found that the optimal cost is at minimal when there are no shortages, which is an expected result. It is obvious that the higher the shortages, the higher the cost of the shipment. This may lead to the high cost of the goods on the final consumers.

\section{Conclusion}

We have considered distribution of goods with stochastic shortages from factories to stores. This paper modeled the distribution of goods from factories to stores in the presence of stochastic shortages of the goods in transit, and the deterministic case (i.e., when there are no shortages) was also considered, using a dynamic optimization technique. Numerical determination of optimal cost and optimal control policy for the operations were obtained. We obtained the optimal transportation cost of shipping goods from factories to stores. The optimal control policies were also obtained. We found that the optimal costs of the goods recovered 
could be determined. It was found that the optimum costs of distributing the goods with minimum and maximum error bounds coincide only at infinity. We referred to this point as a "point of harmony."

\section{References}

[1] C. R. Nwozo and C. I. Nkeki, "On distribution of goods using dynamic programming principles with error bounds," Journal of Applied Mathematics and Bioinformatics, vol. 2, no. 1, pp. 1-19, 2012.

[2] W. B. Powell, "A comparative review of alternative algorithms for the dynamic vehicle allocation problem," in Vehicle Routing: Methods and Studies, B. Golden and A. Assad, Eds., pp. 249-292, North-Holland, Amsterdam, The Netherlands, 1988.

[3] R. K. Cheung and W. B. Powell, "AN algorithm for multistage dynamic networks with random arc capacities, with an application to dynamic fleet management," Operations Research, vol. 44, no. 6, pp. 951-963, 1996.

[4] G. A. Godfrey and W. B. Powell, "An adaptive dynamic programming algorithm for dynamic fleet management, I: single period travel times," Transportation Science, vol. 36, no. 1, pp. 21-39, 2002.

[5] B. van Roy, D. P. Bertsekas, Y. Lee, and J. N. Tsitsiklis, "A neuro-dynamic programming approach to retailer inventory management," in Proceedings of the 36th IEEE Conference on Decision and Control, pp. 4052-4057, December 1997.

[6] J. M. Mulvey and H. Vladimirou, "Stochastic network programming for financial planning problems," Management Science, vol. 38, no. 11, pp. 1642-1664, 1992.

[7] J. M. Mulvey and A. J. Ruszczynski, "A new scenario decomposition method for large-scale stochastic optimization," Operations Research, vol. 43, no. 3, pp. 477-490, 1995.

[8] W. B. Powell, A. Ruszczynski, and H. Topaloglu, "Learning algorithms for separable approximations of stochastic optimization problems," Tech. Rep., Department of Operations Research and Financial Engineering, Princeton University, 2002.

[9] W. B. Powell and H. Topaloglu, "Stochastic programming in transportation and logistics," in Handbook in Operation Research and Management Science, A. Ruszczynski and A. Shapiro, Eds., pp. 249-292, Elsevier, Amsterdam, The Netherlands, 2003, Volume on Stochastic Programming.

[10] B. W. Powell and B. van Roy, "Approximate dynamic programming for high-dimensional resource allocation problems," in Handbook of Learning and Approximate Dynamic Programming, IEEE Press, New York, NY, USA, 2003.

[11] C. Guestrin, D. Koller, and R. Parr, "Efficient solution algorithms for factored MDPs," Tech. Rep., 2003.

[12] M. Z. Spivey and W. B. Powell, "The dynamic assignment problem," Transportation Science, vol. 38, no. 4, pp. 399-419, 2004.

[13] R. Cogill, M. Rotkowitz, B. van Roy, and S. Lall, An Approximate Dynamic Programming Approach to Decentralized Control of Stochastic Systems, Stanford University Press, 2004.

[14] H. Topaloglu and S. Kunnumkal, "Approximate dynamic programming methods for an inventory allocation problem under uncertainty," Naval Research Logistics, vol. 53, no. 8, pp. 822-841, 2006.

[15] M. Hauskrecht and T. Singliar, "Monte-Carlo optimizations for resource allocation problems in stochastic network systems," http://arxiv.org/abs/1212.2481.
[16] K. Ozbay, W. Xiao, C. Iyigun, and M. Baykal-Gursoy, "Probabilities programming models for dispatching and resource allocation in traffic incident management," 2004, http://ie.rutgers .edu/resource/research_paper/paper_05-025.pdf.

[17] P. Jacko, "Stochastic programming framework for resource allocation and (nearly-) optimal priority rules," BCAM-EHU/UPVRobotiker Joint Workshop, 2009.

[18] S. E. Elmaghraby and G. Ramachandra, "Optimal resource allocation in activity networks under stochastic conditions," 2009, http://www.ise.ncsu.edu/people/faculty/docs/elmaghrabyPaper.pdf.

[19] C. R. Nwozo and C. I. Nkeki, "On dynamic optimization technique for resource allocation problems in a transportation network," Journal of Mathematical Association of Nigeria, vol. 36, pp. 30-37, 2009.

[20] C. I. Nkeki, "The use of dynamic optimization technique for the allocation of buses from different stations to different routes by a transportation company in Nigeria," International Journal of Computational and Applied Mathematics, vol. 4, pp. 141-152, 2009.

[21] C. R. Nwozo and C. I. Nkeki, "On a dynamic optimization technique for resource allocation problems in a production company," American Journal of Operations Research, vol. 2, pp. 357-363, 2012.

[22] W. B. Powell, Approximate Dynamic Programming For Asset Management, Princeton University Press, Princeton, NJ, USA, 2004. 


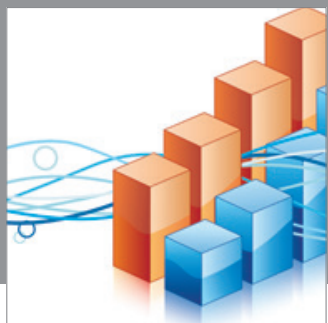

Advances in

Operations Research

mansans

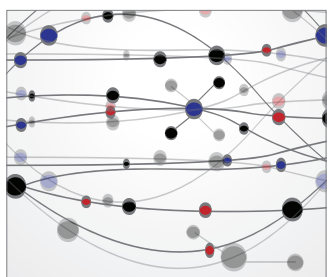

The Scientific World Journal
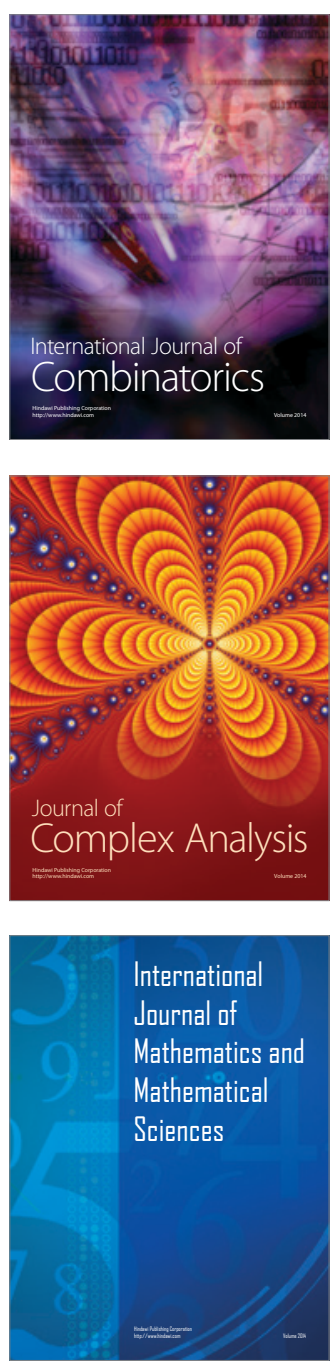
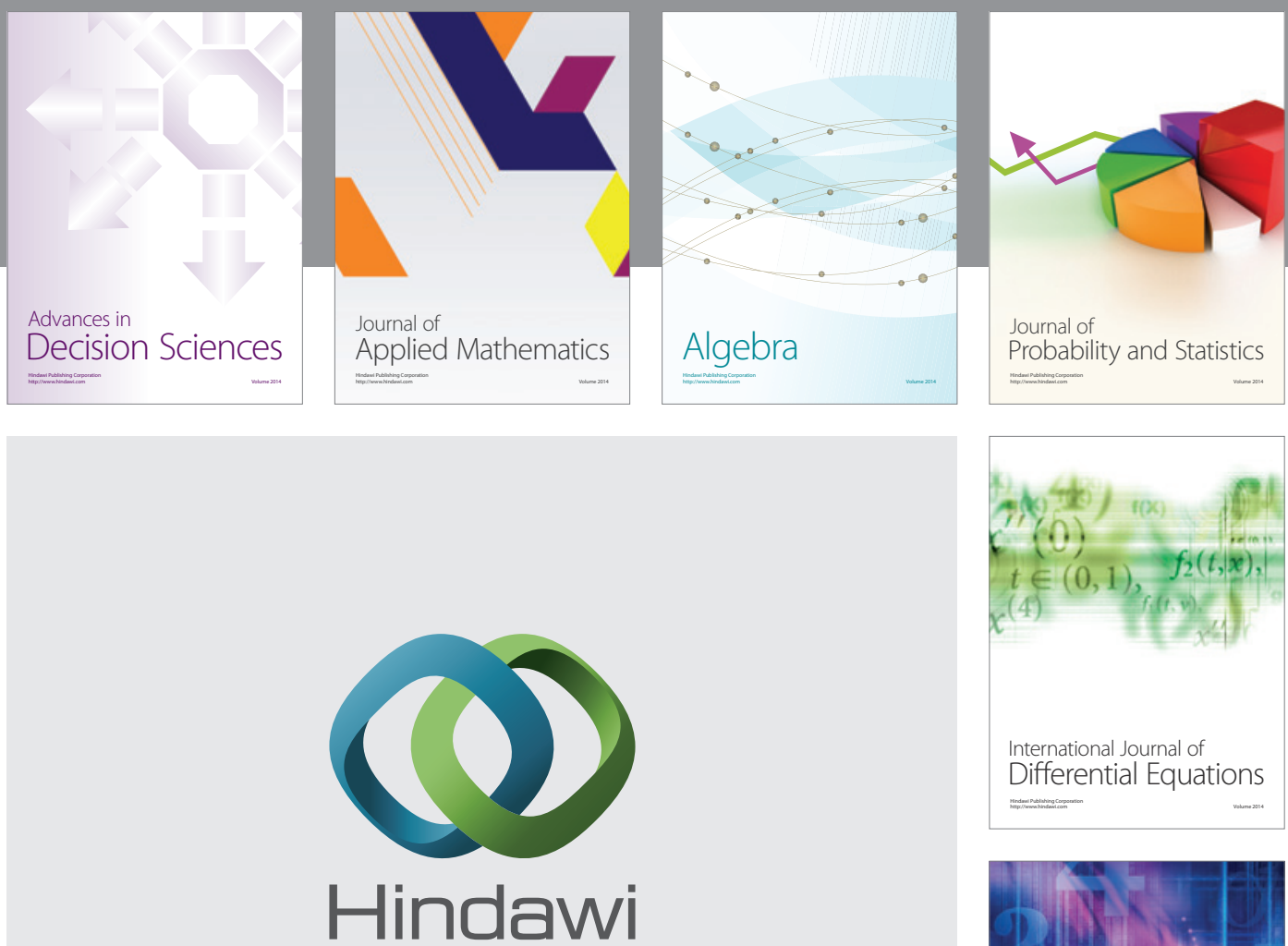

Submit your manuscripts at http://www.hindawi.com
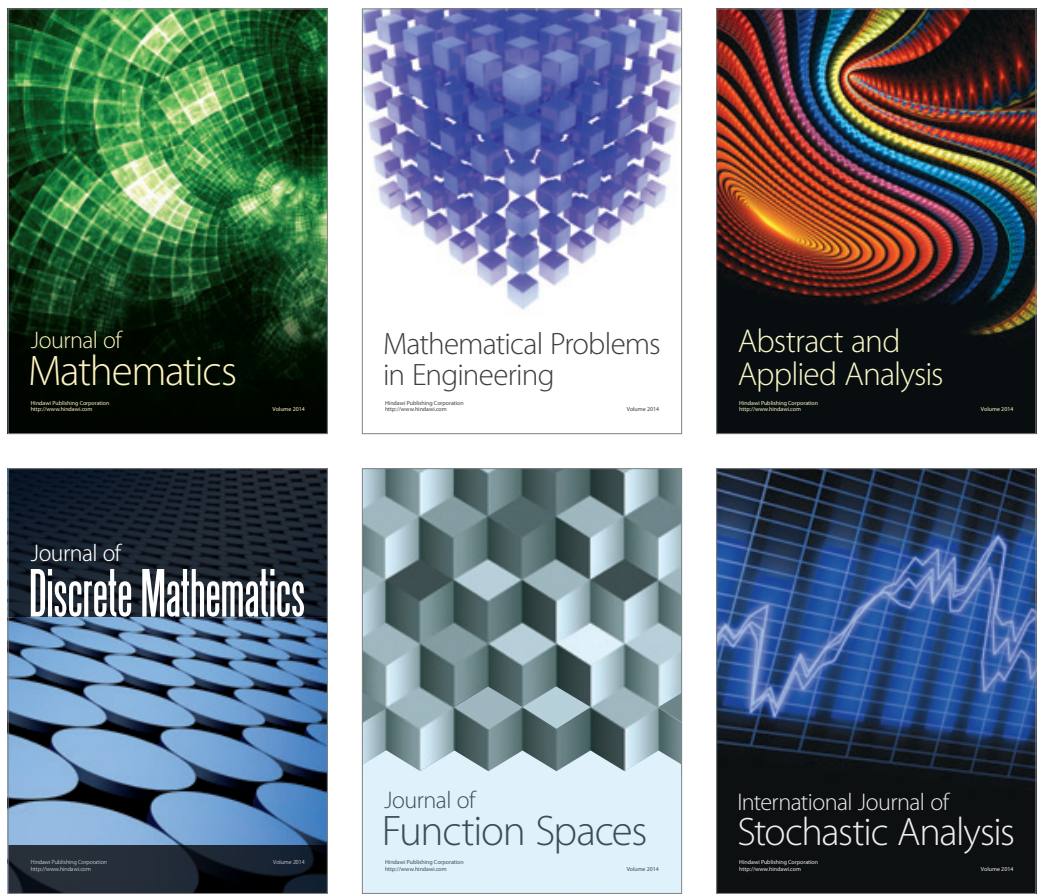

Journal of

Function Spaces

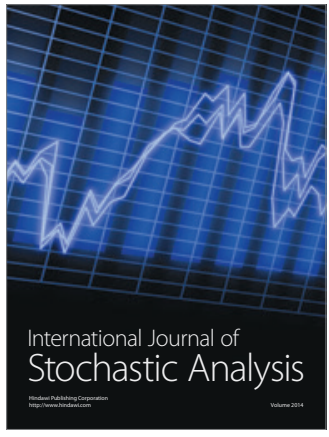

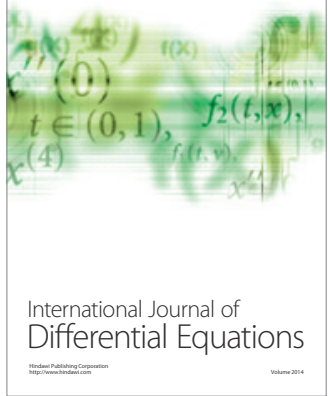
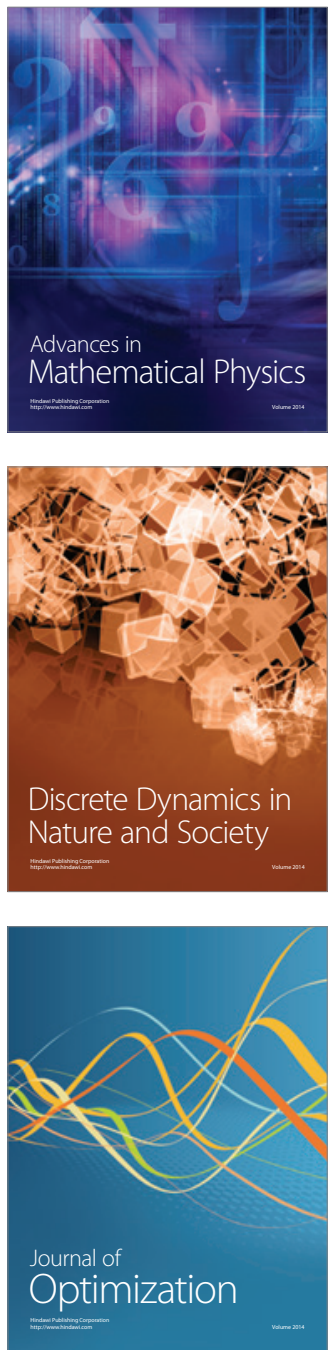